\title{
The use of intraoperative graft assessment in guiding graft revision
}

\author{
Teresa M. Kieser ${ }^{1}$, David P. Taggart ${ }^{2}$ \\ ${ }^{1}$ Department of Cardiac Sciences, Libin Cardiovascular Institute of Alberta, University of Calgary, Calgary, Canada; ${ }^{2}$ Nuffield Department of \\ Surgery, University of Oxford, John Radcliffe Hospital, Oxford, UK \\ Correspondence to: Teresa M. Kieser, MD, PhD. Rm C814, Foothills Medical Centre, $140329^{\text {th }}$ St NW, Calgary, Alberta T2N 2T9, Canada. \\ Email: t.kieserprieur@ucalgary.ca.
}

\begin{abstract}
Quality assurance (QA) in medicine is the practice of the prevention of errors and avoiding problems when delivering care in the form of medical therapy, both in terms of non-invasive and invasive procedures. It is rightly expected by patients. Up until the last 10 years, verification of intraoperative bypass graft patency was limited to a stable hemodynamic status, lack of electrocardiographic evidence of myocardial infarction and, if available, no new regional wall motion abnormalities on transesophageal echo. This perspective outlines two technologies for QA during coronary artery bypass graft (CABG) surgery: transit-time flow measurement (TTFM) for functional assessment of coronary grafts and anatomical evaluation with epicardial ultrasound (ECUS). TTFM is a seasoned technology, used since the late 1990s. ECUS is relatively new, used since 2012. TTFM alone, although useful for intraoperative bypass graft assessment, is not enough; 10-15\% of graft values are ambiguous as to the efficacy of graft function. Therefore, although newer, ECUS is already being established as an indispensable tool for quality assessment in coronary surgery. The two modalities combined are vital for 'state of the art' intraoperative bypass graft assessment.
\end{abstract}

Keywords: Transit-time flow; epicardial ultrasound (ECUS); quality assurance (QA)

Submitted Jun 15, 2018. Accepted for publication Jul 23, 2018.

doi: 10.21037/acs.2018.07.06

View this article at: http://dx.doi.org/10.21037/acs.2018.07.06

\section{Introduction}

The coronary artery bypass graft (CABG) surgery procedure, now 54 years old since Vasilii Kolesov performed the first internal mammary artery-coronary artery anastomosis on Feb 25, 1964, has stood the test of time (1). This is despite the many different types of intracoronary stents used for percutaneous coronary intervention, many of which have been heralded as a possible substitute for CABG. This operation has progressed from all vein grafts [1961-1986] to one-internal-thoracic-artery + saphenous-vein grafts [19861998] (2), to the now ever-increasingly popular multiple arterial graft CABGs (1998-present) (3). Importantly, quality assurance $(\mathrm{QA})$ of the $\mathrm{CABG}$ procedure is receiving attention for multiple reasons: (I) failed bypass grafts may exist without any intraoperative sign, including hemodynamic instability, electrocardiographic signs of myocardial infarction and/ or new regional wall abnormalities seen on transesophageal echocardiography (4). (II) Transit-time flow measurement
(TTFM) and epicardial ultrasound (ECUS) are reliable intraoperative graft patency validation techniques (5). (III) It is prudent to verify connections between two 1-2 mm vessels (5). (IV) Intraoperative QA techniques have long existed for other cardiac operations, such as intraoperative transesophageal echocardiography for mitral valve repair (6). These two QA intraoperative techniques have been developed specifically for assessment of patency of CABGs and represent a change in the culture of CABG. They should be used to improve patient outcomes $(7,8)$. However, 'change' may not always be readily embraced. This perspective outlines the use of TTFM and ECUS for comprehensive QA of CABG.

\section{TTFM}

Walpoth et al. in 1998 first reported the use of TTFM to detect graft failure intraoperatively (9). In 2010 and 2014, The European Guidelines on Myocardial Revascularization 


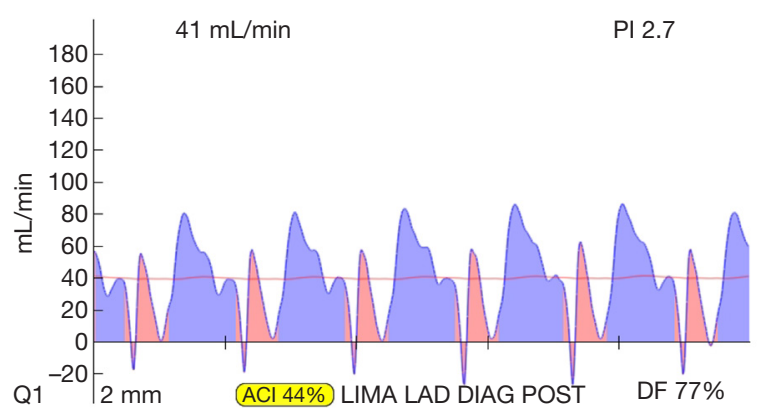

Figure 1 TTFM tracing of an acceptable sequential LIMAdiagonal-LAD graft, post protamine. Flow is $41 \mathrm{~mL} / \mathrm{min}, \mathrm{PI}$ is 2.7 and DF is $77 \%$. TTFM, transit-time flow measurement; LIMA, left internal mammary artery; LAD, left anterior descending; PI, pulsatility index; DF, diastolic filling.

$(7,8)$ recommended the use of TTFM to correct imperfect bypass grafts while still in the operating room, preventing graft failure and potential patient morbidity and death. However, in 2017 only $30 \%$ of the 800,000 CABG procedures world-wide annually were assessed for graft patency using TTFM*.

The basics of TTFM are easy to learn. Parameters for acceptable bypass graft function are: flow $>15 \mathrm{c} / \mathrm{min}$, pulsatility index $(\mathrm{PI})<5.0$ for acceptable graft; $<3.0$ for ideal value and diastolic filling $60-80 \%$ for left-sided vessels and $45-55 \%$ for right sided vessels (Figure 1). However, with continued routine use, one begins to appreciate the nuances of coronary flow, such as flow competition, how to detect false negative results and the subtle changes of less-thanperfect but functioning grafts (6). Multiple measurements at various stages of the bypass operation will allow identification of a suboptimal graft at a point in time when revision will minimally impact operative time, patient morbidity and surgical team/surgeon frustration. Measurement times for off-pump include: post completion of the anastomosis with the stabilizer in place, with a proximal snare of the native coronary (if a snare is used), without the stabilizer, with the heart and patient in the neutral position and after protamine is administered. On-pump CABG measurement times include: on cross-clamp, on-pump without cross-clamp, offpump and post protamine administration.

Most often, the first value for both off- and onpump is a measure of the adequacy of the conduit and distal anastomosis. Subsequent values bring into play other problematic aspects, such as the length, lie, or
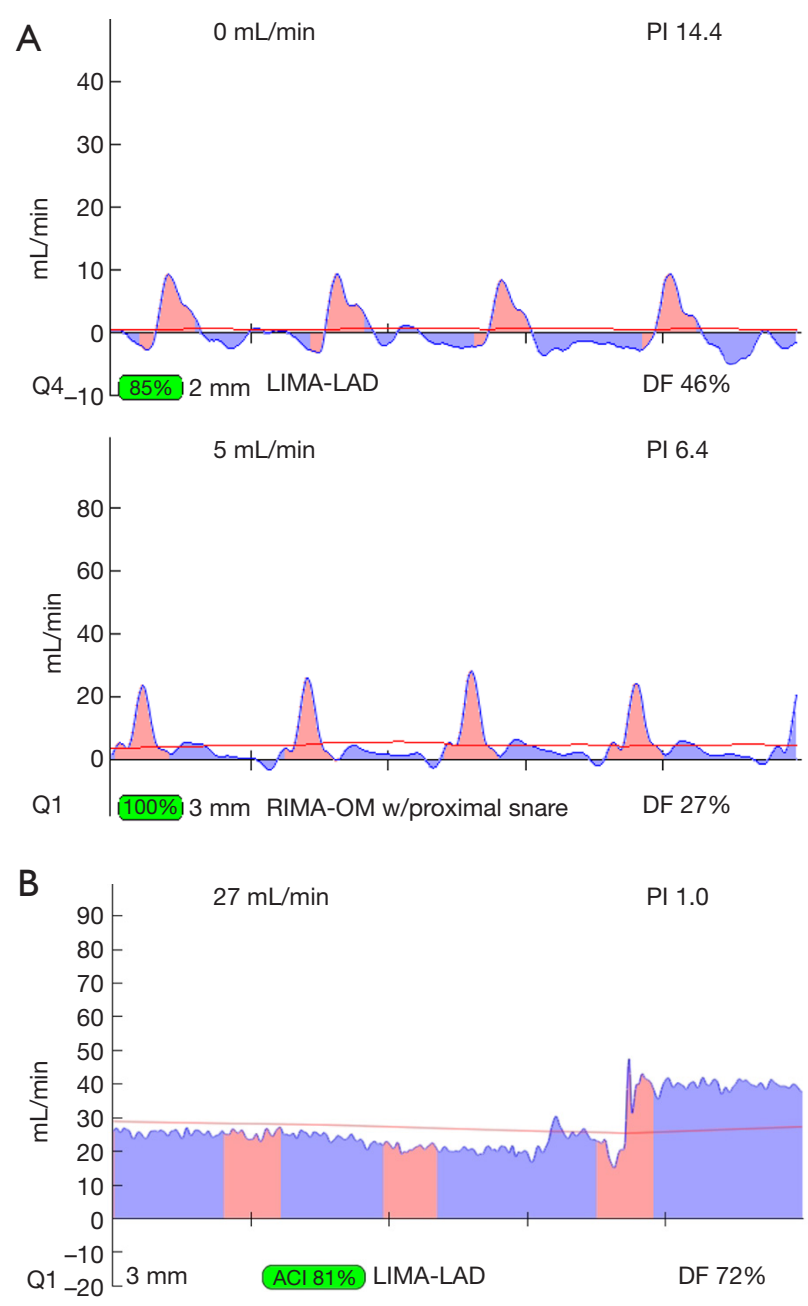

Figure 2 Transit-time flow measurements of occluded grafts (first two tracings) and the importance of correct probe position on a bypass graft (third tracing). (A) Two examples of 'true positives' or occluded grafts with predominant pink spikes of systolic flow and minimal blue waves below the baseline indicating little/no diastolic flow. (B) Example of the mechanism of a 'false positive' graft. This reading has caught on screen the changing angle of the probe: on the left side of the image, the probe is not at right angles with the graft and shows lower flow, but when the angle is corrected (right side of image) the flow rises and the PI is good. PI, pulsatility index.

configurations of the conduit. TTFM values with low flow and high PI accurately predict the likelihood of graft occlusion (the 'true positive') (Figure $2 A$ ). A false positive (i.e., a good graft with high PI) rarely occurs and is most

\footnotetext{
* Personal communication from Mr. Roger Morberg, VP Sales International, Medistim ASA, Oslo, Norway Sept 12, 2017.
} 
often due to improper probe placement or competitive flow (Figure 2B). The specificity of TTFM (i.e., the ability to predict a good graft, or 'true negative') is $98.4 \%$ (10) (Figure 3). The only caveat of TTFM is the false negative, that is, an imperfect graft with a falsely-low PI. This usually occurs when flow is retrograde in the coronary artery and is obstructed at the toe (Figure 4). TTFM Examples are shown in Figure $5 A, B, C$. One of the most common reasons for graft imperfection is the placement of extra sutures for hemostasis; it is prudent to measure flow after each repair stitch (Figure 6). One can also learn of arterial graft's ability to increase flow by checking TTFM at re-operation for bleeding in the subsequent post-op hours or days (Figure $7 A, B$ ).

For most accurate graft flows, it is best to measure flows near as possible to the distal anastomosis, as this is more likely to reflect the flow into the coronary

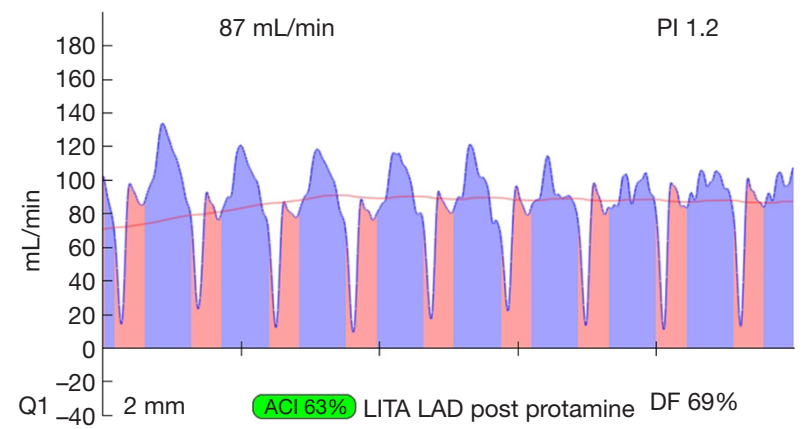

Figure 3 Example of a 'true negative' LIMA-LAD graft, this is a good graft with flow $>15 \mathrm{cc} / \mathrm{min}$ and PI <3.0. LIMA, left internal mammary artery; LAD, left anterior descending artery; PI, pulsatility index. bed. Measurement far from the distal anastomosis also incorporates the resistance conferred by the length of the conduit. However, for a marginal artery graft on the posterior surface of the heart, after off-pump, this may not be possible. In this instance, measurements are taken where convenient for non-displacement of the heart, near the aortic origin of a free graft or near the inflow as for the proximal RIMA travelling through the transverse sinus to the circumflex system. However, one must appreciate that measurement at the 'far end' of a graft may have systemic arterial pressure aspects with wave forms reflective of this systemic pressure (i.e., more equal diastolic and systolic filling and/or backward flow). To this point, one subtle and not commonly appreciated facet is the difference in TTFM values which can occur at various locations along the conduit. This most often occurs in in situ right internal mammary artery (RIMA) grafts to the right coronary artery (RCA) system. An in situ IMA artery at the proximal end is still attached to the systemic circulation, which fills its target organs during systole. TTFM of a systemic artery shows mostly a pink systolic spike, which is normal for a systemic artery. Figure 8 shows the TTFM of a harvested IMA still functioning as a systemic artery attached to the chest wall (Figure 8). Note that the normal pink systolic spikes are similar to the waveform of an occluded bypass graft. This is why it is important to realize that measuring an in situ RIMA at different locations along the conduit may result in three different waveforms; at proximal, mid and distal locations along the conduit, it is possible to see the progression of the RIMA graft waveform as it starts off as a systemic artery filling mostly in systole and progresses to being a coronary artery filling the heart mostly in diastole (Figure 9A,B,C). The authors have noted that this phenomenon dissipates the longer the IMA is attached, as

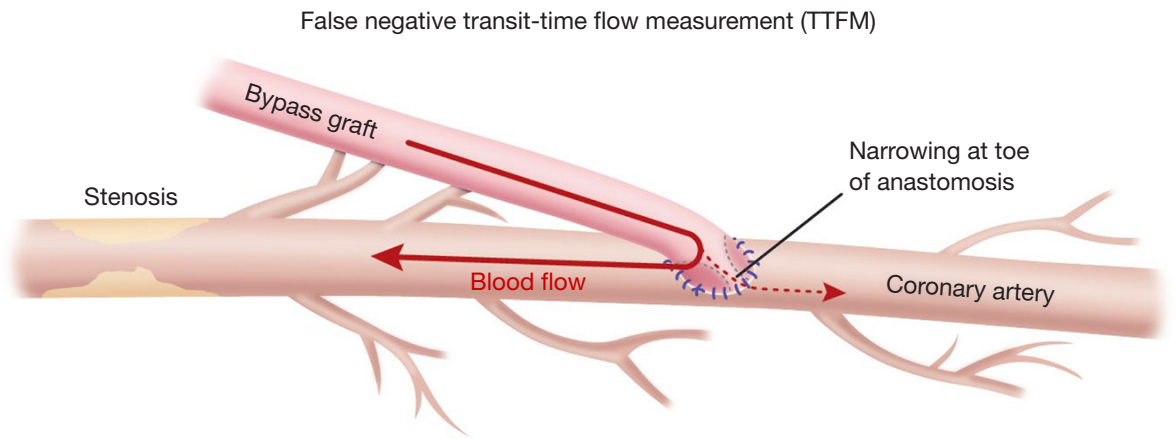

Figure 4 Illustration of one possible cause of 'false negative' measurement for TTFM. This is most likely to occur in left anterior descending bypass grafts because the myocardium is supplied both anterograde and retrograde to the anastomosis with the rich network of septal perforators. TTFM, transit-time flow measurement. 

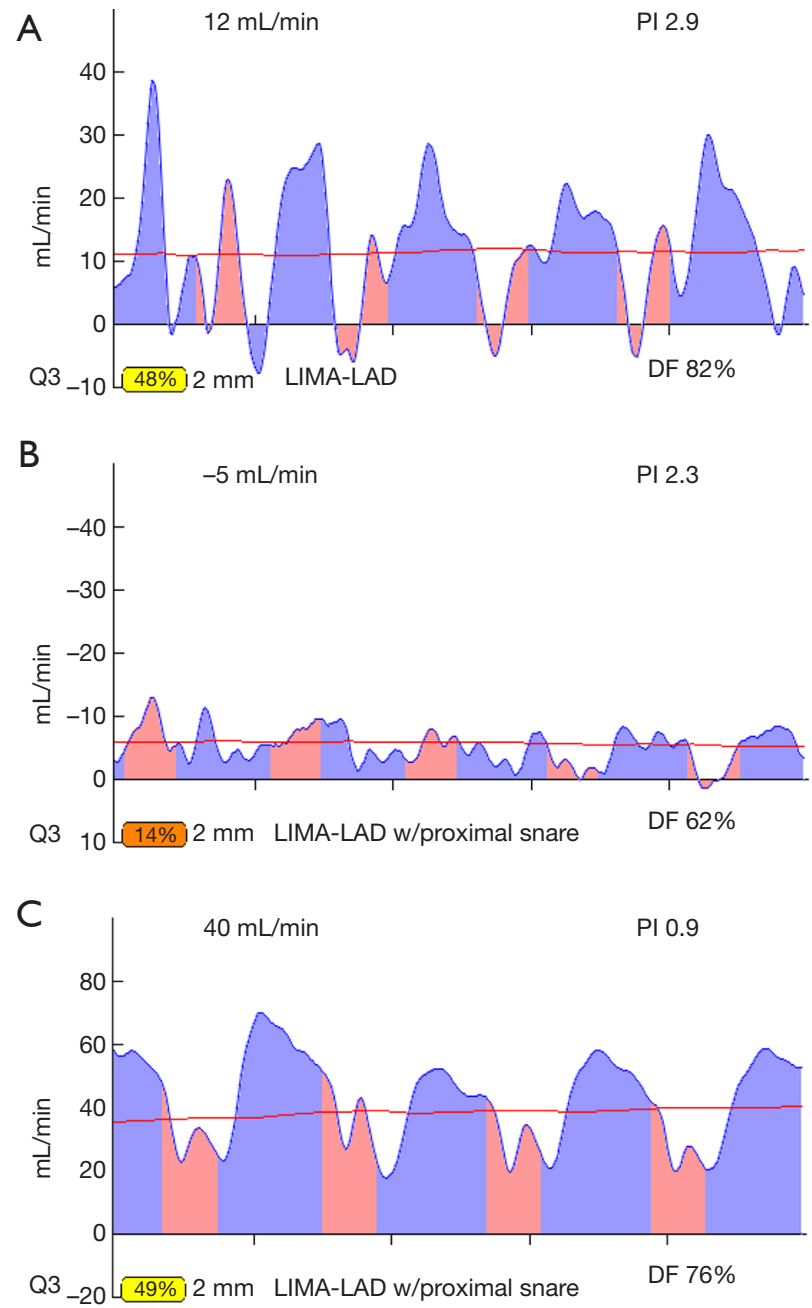

Figure 5 Transit-time flow measurement of a suboptimal graft manifest only when snaring of proximal coronary artery. (A) TTFM of seemingly acceptable LIMA-LAD graft according to PI, but flow is sub-optimal. (B) With a proximal snare in place on the native coronary artery, the flow is reduced, indicating a problem likely at the toe of the graft. (C) After graft revision, the wave form shows heightened flow and very low PI, indicative of a previously ischemic coronary bed due to ischemic vasodilation. When this flow pattern is seen after graft revision, this graft was not satisfactory, even if no reason for error was found at time of revision. PI, pulsatility index; TTFM, transit-time flow measurement; LIMA, left internal mammary artery; LAD, left anterior descending.

the operation progresses. This phenomenon may not persist at the end of the operation, prior to closing the chest. This is just one example of the many nuances of TTFM that can be appreciated with practiced use of this technique of
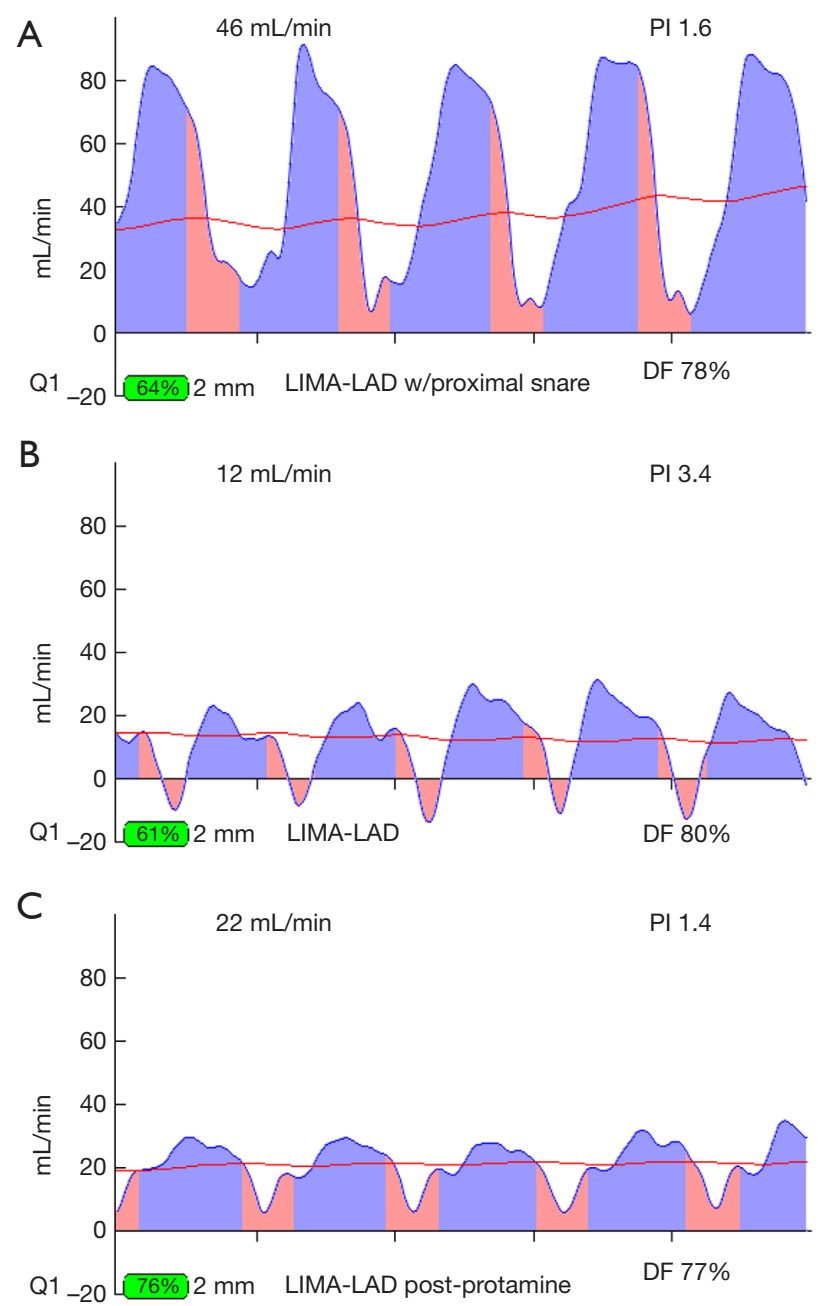

Figure 6 Examples of how placement of an extra stitch may cause a graft problem. (A) Graft with a leak; (B) graft with extra stitch; (C) extra stitch removed and replaced with more accuracy.

intraoperative graft assessment.

In these authors' experience, in $10-15 \%$ of TTFM measurements, the readings are difficult to interpret or ambiguous as to whether the graft is functioning properly. Several authors have also published concerns regarding the accuracy of TTFM alone (6,8,11-14). For complete intraoperative graft assessment, ECUS is needed; ECUS allows anatomical verification of a good bypass graft, in addition to the functional validation with TTFM.

\section{ECUS}

The discovery and perfection of current ECUS for 

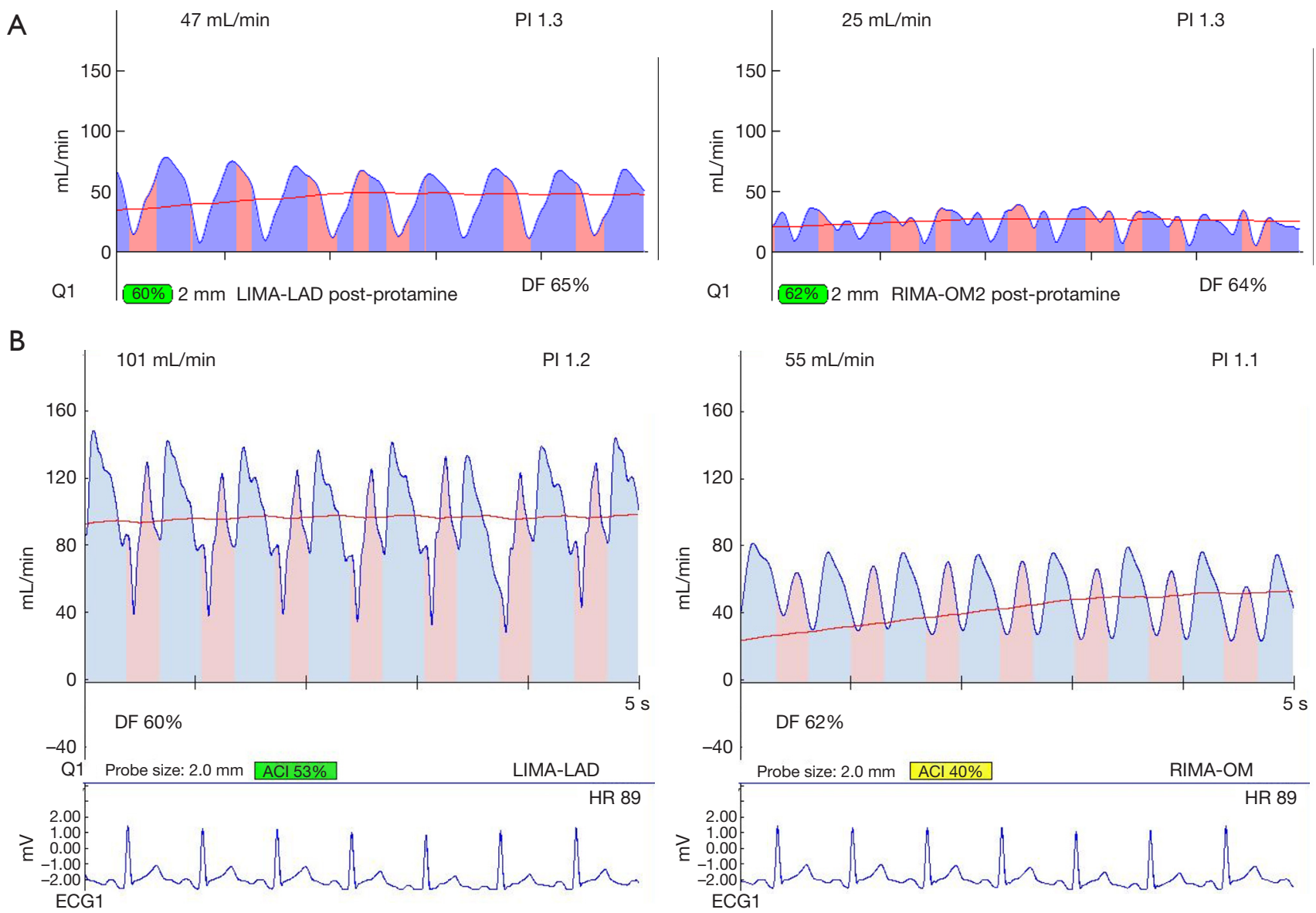

Figure 7 Example of TTFM values 24 hours postoperatively at re-operation for bleeding. (A) TTFM at time of initial surgery of LIMA and RIMA; (B) TTFM LIMA and RIMA at re-operation 24 hours later. LIMA, left internal mammary artery; RIMA, right internal mammary artery; TTFM, transit-time flow measurement.

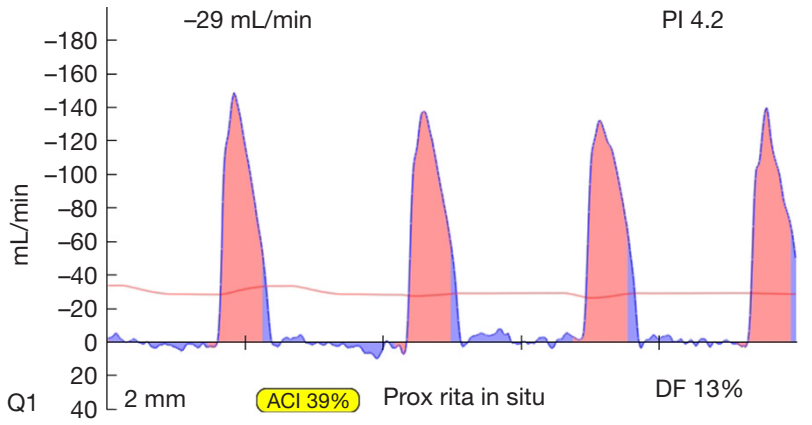

Figure 8 TTFM of harvested in situ right internal mammary artery (RIMA) before transection from the chest wall. The RIMA is still a systemic artery shown by the pink systolic spikes and minimal filling in diastole (blue wave forms). When anastomosed to a coronary artery this would indicate an occluded graft. TTFM, transit-time flow measurement. intraoperative bypass graft assessment took over 30 years. Intraoperative ECUS of coronary artery anastomoses was first reported by Hiratzka et al. in 1986 (15). Prior to then, previous ECUS reports by cardiologists in 1985 had concentrated on in vivo and in vitro coronary artery similarities with angiography (16). Seventeen years later, in 2002, Haaverstad et al. used a perfected, sterilizable probe with epicardial color Doppler ultrasound to demonstrate accurate assessment of 23 LIMA-LAD anastomoses on the beating heart (17). Then in 2012, at the European Association of Cardio-thoracic Surgery Annual Meeting in Barcelona, Medistim ASA introduced the VeriQC machine, with imagery that, for the first time, incorporated TTFM with ECUS using a re-sterilizable ECUS probe. The latest reiteration of this device is a multiple component device- 


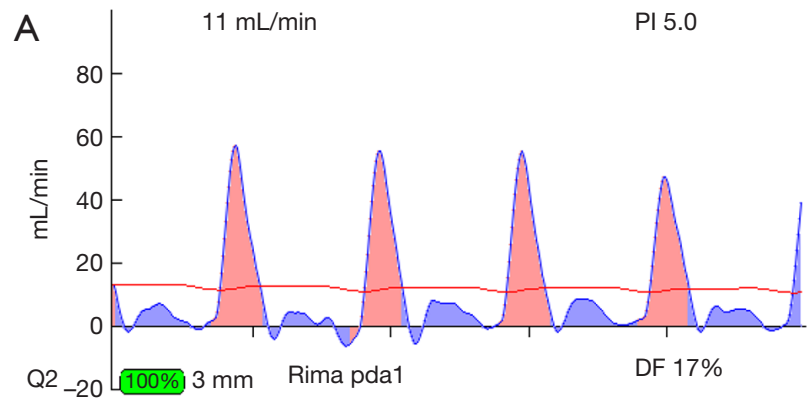

B
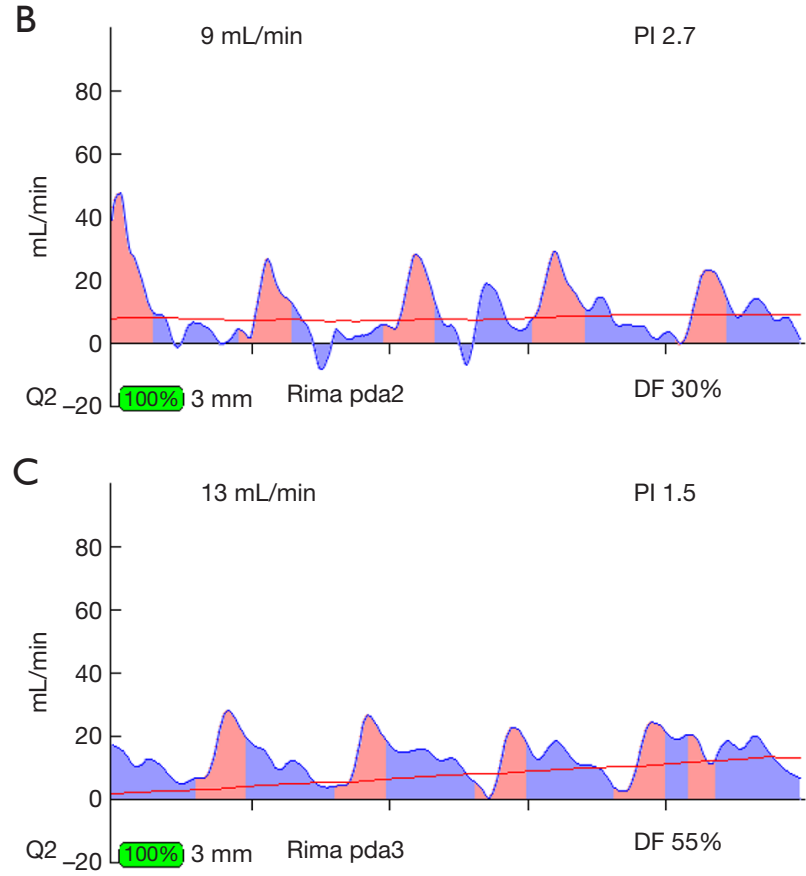

Figure 9 Different locations of TTFMs on RIMA conduit of RIMA to PDA graft. (A) TTFM of RIMA to PDA close to the proximal end near the subclavian artery. Note the predominant pink spike, as the RIMA in this location is proximate to the systemic circulation. (B) TTFM of the same RIMA to PDA at the midpoint of the conduit. Note that there is more filling in diastole than at the proximal end of the conduit. (C) TTFM of the same RIMA to PDA taken at the distal end of the conduit just ahead of the distal anastomosis. Note that the majority of the filling is in diastole. TTFM, transit-time flow measurement; RIMA, right internal mammary artery; PDA, posterior descending coronary artery.

the Medistim MiraQ system. There is as well another epicardial ultrasound probe: the Philips L15-7io linear array ultrasound probe of the iE33 Philips transesophageal machine. This ECUS probe is not sterilizable and must be used in a sterile sleeve.

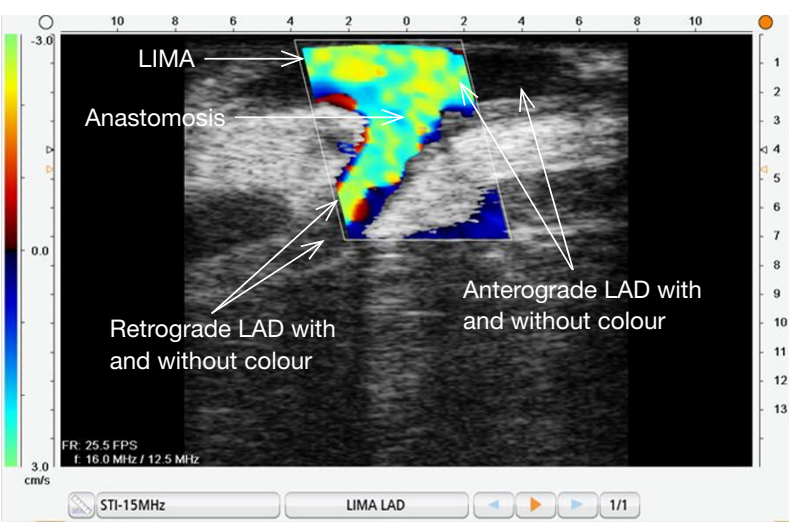

Figure 10 Longitudinal epicardial ultrasound of LIMA-LAD. Look outside the colour to see the anterograde and retrograde native coronary artery (ECUS image by Medistim MiraQ system). LIMA, left internal mammary artery; LAD, left anterior descending.

ECUS assessment of distal anastomoses is akin to performing a color angiogram intraoperatively at a time when a bypass graft can be revised at minimal cost to the patient. Figure 10 shows a LIMA-LAD anastomosis in the longitudinal dimension. All locations of anastomoses may be assessed with ECUS. Proximal and distal anastomoses may be verified to be patent, as seen in Figure 11. The technique of using ECUS for off-pump is best performed with the coronary stabilizer still in place; performing ultrasound on a moving target can actually be more difficult than performing the anastomosis itself. For on-pump CABG, imaging is performed just after completion of the distal anastomosis with the cross-clamp still on. A good ECUS examination of a distal anastomosis supersedes an equivocal TTFM value. Di Giammarco found that with the addition of ECUS to TTFM, the positive predictive value (PPV) of the two modalities together approached 100\% (14). With use of TTFM alone, although sensitivity (i.e., the true positive rate) varies from $94-98 \%$, specificity (i.e., the true negative rate) is much lower at $61 \%(10,14)$.

ECUS has many more uses than just validation of a patent anastomosis. It can be used to evaluate a conduit pre- and post-harvest for detection of an ITA dissection (Figure 12) (18), the ascending aorta for soft non-palpable atherosclerotic plaque (Figure 13), or absence of aortic dissection after de-cannulation of the aortic cannula (Figure 14). The native coronary arteries can be assessed by ECUS for determination of the optimal site for anastomosis (Figure 15A,B). ECUS can also determine the depth of a coronary artery below the epicardium, that is, the determination of an in intramyocardial artery (Figure 16). 

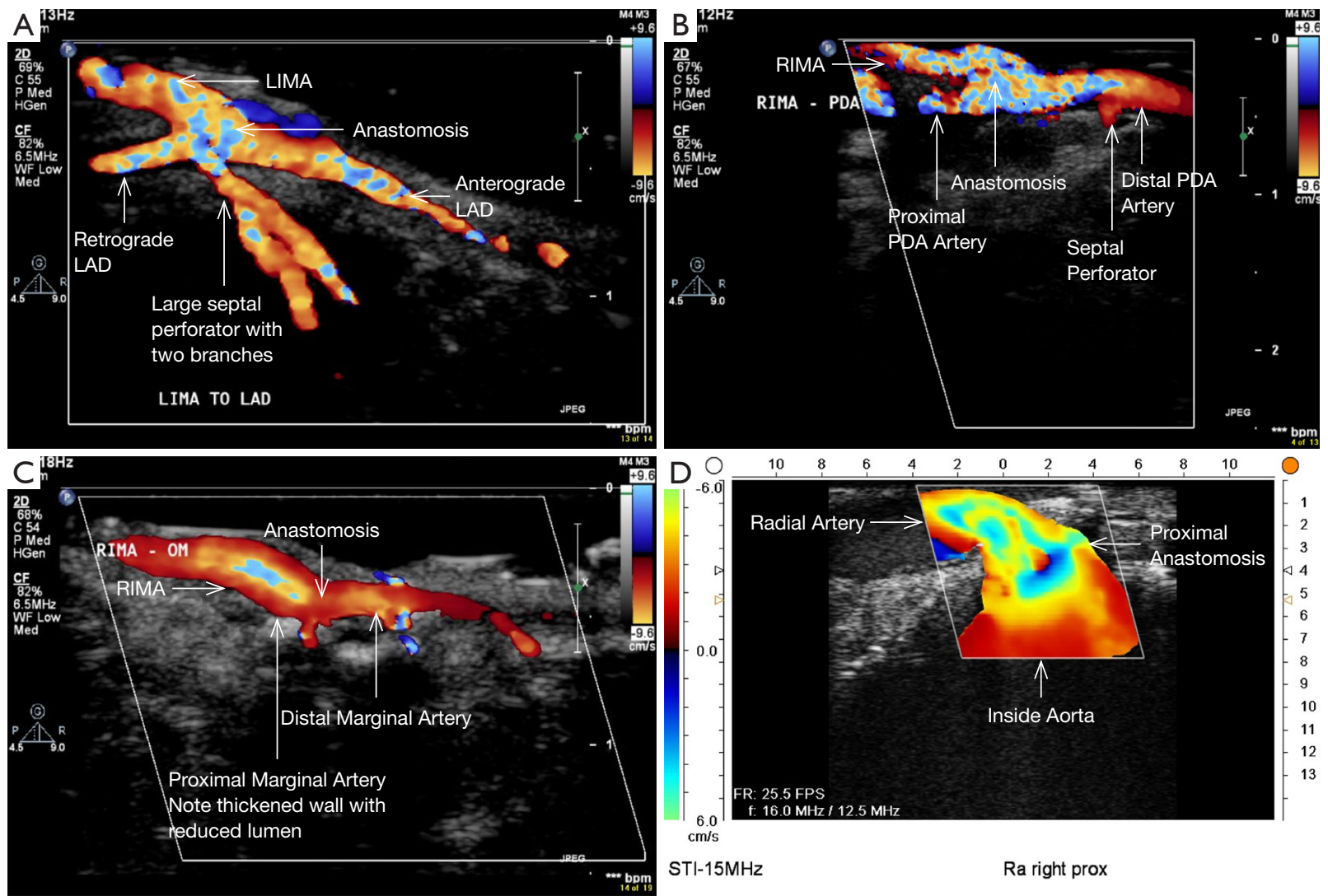

Figure 11 Intraoperative ECUS images of bypass grafts. (A) ECUS of LIMA to LAD with septal perforators; (B) ECUS of RIMA-PDA as in situ graft; (C) ECUS of RIMA-marginal artery through the transverse sinus as in situ graft; (D) ECUS of proximal anastomosis of Radial artery to right coronary artery [ECUS images (A,B,C) by ECUS probe L15-7io of iE33 Philips transesophageal machine; ECUS image (D) by Medistim MiraQ system]. ECUS, epicardial ultrasound; LIMA, left internal mammary artery; LAD, left anterior descending; RIMA, right internal mammary artery; PDA, posterior descending artery.
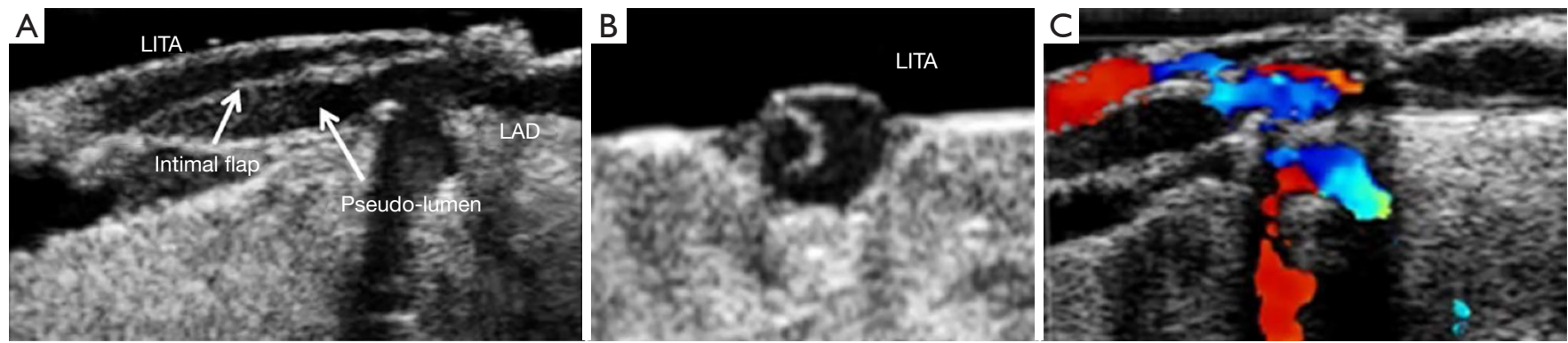

Figure 12 Intraoperative discovery of intimal flap dissection of IMA with ECUS. (A) Longitudinal view; (B) cross-sectional view; (C) Doppler colour flow mapping showing flow restriction by false lumen compressing true lumen [Watanabe, Arai (18)] (ECUS images by Medistim MiraQ system). IMA, internal mammary artery; ECUS, epicardial ultrasound. 

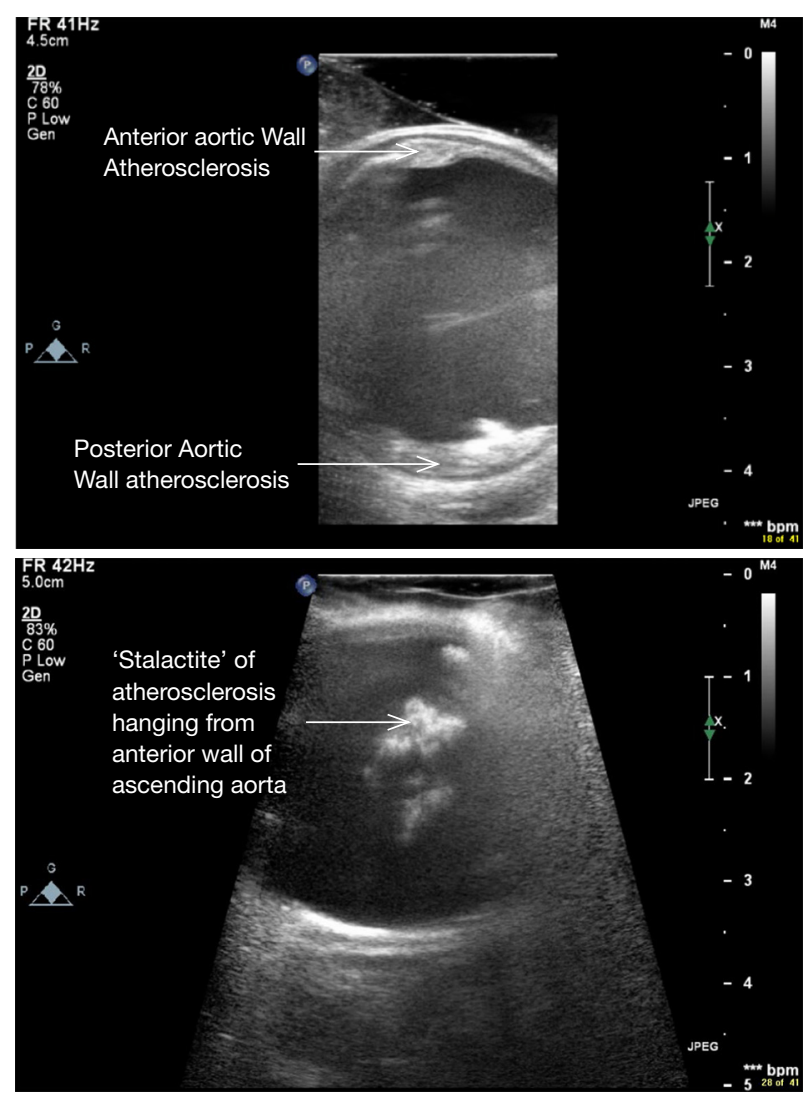

Figure 13 ECUS of ascending aorta in 2 different patients showing prohibitive ascending aortic atherosclerosis, both patients underwent off-pump CABG with no stroke (ECUS images by Medistim MiraQ system). ECUS, epicardial ultrasound; CABG, coronary artery bypass graft.

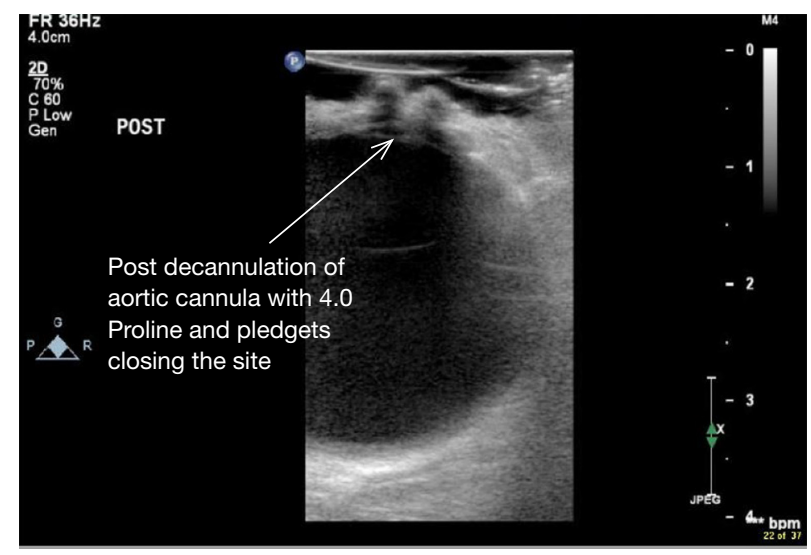

Figure 14 ECUS of ascending aorta after de-cannulation of aortic cannula to check for absence of aortic dissection (ECUS images by Medistim MiraQ system). ECUS, epicardial ultrasound.

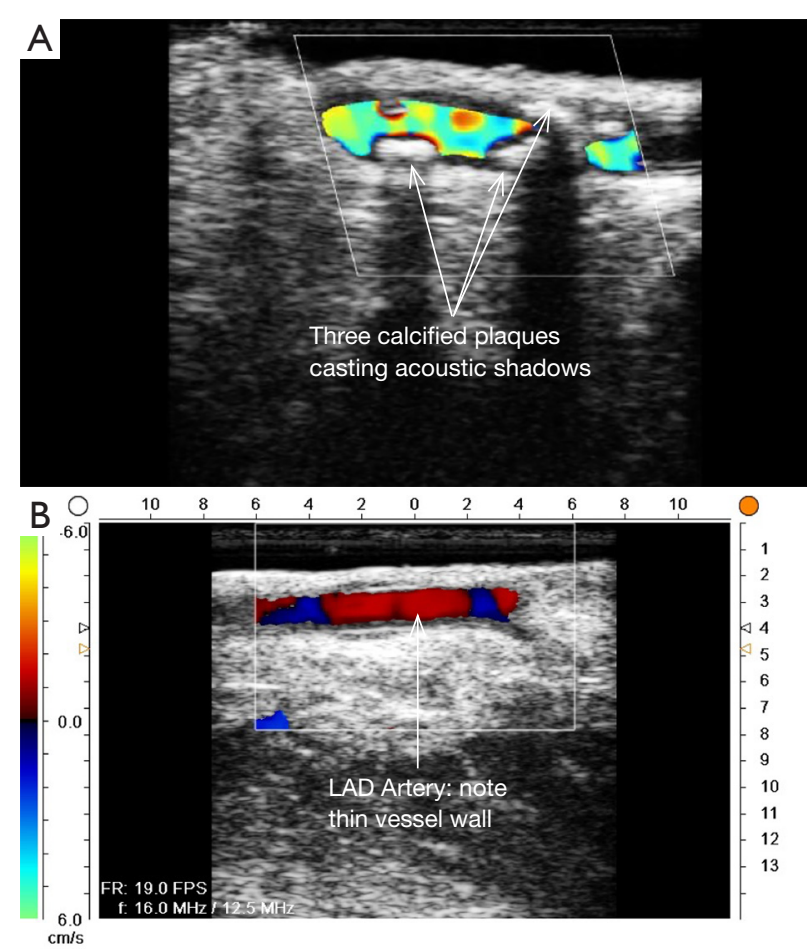

STI-15MHz

LAD

Figure 15 ECUS of native coronary arteries for determination of best anastomotic site. (A) ECUS of diseased LAD with multiple calcification areas causing acoustic shadows. (B) ECUS of LAD artery in a good location to perform distal anastomosis (ECUS images by Medistim MiraQ system). ECUS, epicardial ultrasound; LAD, left anterior descending artery.

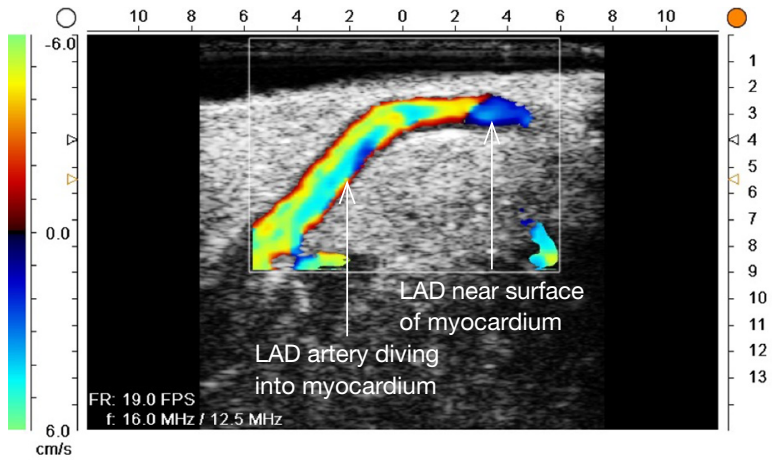

STI-15MHz

LAD

Figure 16 ECUS of intramyocardial LAD (ECUS images by Medistim MiraQ system). ECUS, epicardial ultrasound; LAD, left anterior descending artery. 
However, due to the small size of coronary arteries, they can be difficult to locate/use for bypass. Unlike in anesthesia, where an anesthetist uses a 'finder needle' to locate the internal jugular vein, this technique is not possible for coronary artery location. In addition, bypassing a deeply intramyocardial artery carries the risk of inadvertently entering the right ventricle or damaging the LAD by cutting directly through it. Also, in one of the authors experience (Teresa M. Kieser), an anastomosis placed in the first centimetre of the intramyocardial tunnel resulted in graft occlusion 2 months later, requiring reoperation despite excellent TTFM and ECUS values at the first surgery. Knowing that a coronary is intramyocardial serves to let the surgeon know that the coronary must be located proximally or distally to the intramyocardial segment for bypass location.

When tools such as ECUS are available, many uses are discovered serendipitously and/or by necessity. Iino et al. report a suboptimal TTFM reading to have been caused by an 'air lock' inside a RIMA. This was discovered with the use of ECUS on the anastomosis, identifying an occluding bubble of air (19). When chronic, totally occluded coronary arteries are not visible by collateralization on preoperative angiography, operating on these patients can be daunting without the use of ECUS. Inoue et al. report two such patients and found that ECUS not only located both coronaries, but was also helpful in determining (I) the level of the occlusion and (II) the adequacy of the size of the patent lumen for bypass (20).

\section{Perspectives}

Coronary surgery remains the 'gold standard' intervention for most patients with multivessel and/or left main coronary artery disease, but it can always be improved. The use of arterial grafts has been shown in many retrospective reviews to improve quality and/or duration of life. The complete package of 'best practice' CABG surgery includes many techniques not commonly practiced, namely: (I) skeletonization of the IMAs; (II) use of multiple arterial grafts-be they IMAs, radial arteries or gastroepiploic arteries; and (III) intra-operative graft assessment with TTFM and ECUS. Use of the current imaging techniques can, fortunately, start before the other two and be perfected for the common practice of one-IMA + saphenous vein CABG surgery. Once TTFM and ECUS are mastered, the stage is set for progressing to skeletonization of the IMA and then total arterial grafting.

\section{Pros and cons}

Positive aspects of TTFM and ECUS include: (I) fewer failed grafts, which undoubtedly leads to decreased patient morbidity/mortality in both the early and late postoperative phases; (II) self-learning occurs with each and every use of intra-operative graft assessment and includes observing high and low flows, high and low pulsatility indices and their relationship to: size of coronary arteries grafted, level of coronary stenosis, graft configuration and many other indices. Constant learning and the self-assessment that occurs with TTFM and ECUS make surgeons continuously improve; (III) use of TTFM and ECUS at the time of surgery gives a surgeon peace of mind, knowing that everything possible has been done for a patient at the time of the operation when graft revision carries the least patient harm. Post-op telephone calls for the 'failed graft' scenario to a surgeon who routinely uses TTFM and ECUS are few and far between; (IV) good grafts beget good surgery. Good surgery begets a positive cardiologist's mindset toward CABG; as they are responsible for referrals for $\mathrm{CABG}$, more patients are likely to be referred for surgical revascularization if outcomes are increasingly positive. Ultimately, 'positive press' for CABG spirals patient care ever upward.

Negative aspects of use of TTFM and ECUS include: (I) the requirement for surgeons to leave their comfort zone and learn something new. As stated, the basics of TTFM are quite easy and the nuances come gradually, but incessantly. Use of ECUS requires some patience and attention to detail, but the basics can be learned in about 10-15 grafts. ECUS of off-pump bypasses to the lateral and inferior walls may be technically challenging and certainly require the offpump stabilizer for imaging. Cardiac surgery anesthetists, who are well-versed in transesophageal echocardiography, can be an invaluable source of knowledge during the learning curve; (II) there is nothing more humbling than having to revise a graft. However, by not using TTFM and ECUS and re-operating on a patient for a failed graft is not only humbling, but unforgiveable. As said by Kieser, 'To err is buman, to not divine is unforgiveable' (6). Now, with the advent of TTFM and ECUS, this should rarely be necessary; (III) operative time is increased by less than 5 minutes with use of TTFM and by about $1 \frac{1 / 2}{2}$ minutes for each distal anastomosis by use of ECUS. However, the time will be longer if results are equivocal, or if graft revision must be undertaken; (IV) probably what is feared most when surgeons start to use intraoperative graft assessment 
is unnecessary graft revision. There are several reports concerning this problem (13-15). However, the addition of ECUS to TTFM completes the technique of intraoperative graft assessment to near perfection for detection of a suboptimal or occluded anastomosis.

\section{Caveats}

There is one proviso that can negatively affect a surgeon's experience with TTFM and ECUS, and that is, inconsistent use. If a surgeon only uses these modalities when an imperfect graft is suspected, their situation 'will not be improved'. Without constant use of at least TTFM, a false negative may not be suspected, or a false positive may be misinterpreted as a failed graft, leading to unnecessary graft revision. As there is now so much to learn with the addition of ECUS to TTFM, for those never having used either before, the assimilation of knowledge of both modalities simultaneously can be daunting and time consuming while in the operating room. These authors suggest starting first with use of TTFM, use on every graft at least 4 times/graft as suggested above and continue with this modality alone for at least 6 months. In addition, there are now several centres familiar with TTFM and their advice may be sought and also by the company employees who sell the devices. After familiarity is achieved with TTFM, the addition of ECUS will make interpretation of TTFM easier and patients can only benefit from this newly acquired surgical expertise.

\section{Conclusions}

QA for the CABG procedure is now available in the form of TTFM and ECUS. Since the CABG procedure is still one of the most frequently performed major operations in the world, if there are ways to monitor the patency of bypass grafts while the patient is still in the operating room, there is no reason not to use these modalities. TTFM and ECUS help surgeons to see the imperfect graft at a time when revision does the least harm to patients. Surgeons are still human and as such should use these safety nets to ensure their work helps patients through their disease as best as they are able. 'In purity and according to divine law I carry out my life and my art.' (Hippocratic Oath c. 275 AD).

\section{Acknowledgements}

Thank you to all of our patients who entrust to us their present and future cardiac health.

\section{Footnote}

Conflicts of Interest: Prof. Taggart and Dr. Kieser have received research funding, speaking and travelling honoraria from Medistim.

\section{References}

1. Olearchyk AS. Vasilii I. Kolesov. A pioneer of coronary revascularization by internal mammary-coronary artery grafting. J Thorac Cardiovasc Surg 1988;96:13-8.

2. Loop FD, Lytle BW, Cosgrove DM, et al. Influence of the internal-mammary-artery graft on 10-year survival and other cardiac events New Engl J Med 1986;314:1-6.

3. Lytle BW, Blackstone EH, Loop FD, et al. Two internal thoracic artery grafts are better than one. J Thorac Cardiovasc Surg 1999;117:855-72.

4. Kieser TM, Rose S, Kowalewski R, et al. Transit-time flow predicts outcomes in coronary artery bypass graft patients: a series of 1000 consecutive arterial grafts. Eur J Cardiothorac Surg 2010;38:155-62.

5. Kieser TM, Taggart DP. Current status of intraoperative graft assessment: Should I be the standard of care for coronary artery bypass graft surgery? J Card Surg 2018;33:219-28.

6. Kieser TM. Graft quality verification in coronary artery bypass graft surgery: how, when and why? Curr Opin Cardiol 2017;32:722-36.

7. Kolh $\mathrm{P}, \mathrm{Wijns} \mathrm{W}$, Danchin N, et al. Task Force on Myocardial Revascularization of the European Society of Cardiology (ESC) and the European Association for Cardio-Thoracic Surgery (EACTS); European Association for Percutaneous Cardiovascular Interventions (EAPCI), Guidelines on myocardial revascularization. Eur J Cardiothorac Surg 2010;38 Suppl:S1-52.

8. Kolh P, Windecker S, Alfonso F, et al. Task Force on Myocardial Revascularization of the European Society of Cardiology and the European Association for Cardio-Thoracic Surgery; European Association of Percutaneous Cardiovascular Interventions. 2014 ESC/ EACTS Guidelines on myocardial revascularization: the Task Force on Myocardial Revascularization of the European Society of Cardiology (ESC) and the European Association for Cardio-Thoracic Surgery (EACTS). Eur J Cardiothorac Surg 2014;46:517-92.

9. Walpoth BH, Bosshard A, Genyk I, et al. Transit-time 
flow measurement for detection of early graft failure during myocardial revascularization. Ann Thorac Surg 1998;66:1097-100.

10. Desai ND, Miwa S, Kodama D, et al. A randomized comparison of intraoperative indocyanine green angiography and transit-time flow measurement to detect technical errors in coronary bypass grafts. J Thorac Cardiovasc Surg 2006;132:585-94.

11. Nordgaard H, Nordhaug D, Kirkeby-Garstad I, et al. Different graft flow patterns due to competitive flow or stenosis in the coronary anastomosis assessed by transittime flowmetry in a porcine model. Eur J Cardiothorac Surg 2009;36:137-42.

12. Jokinen JJ, Werkkala K, Vainikka T, et al. Clinical value of intra-operative transit-time flow measurement for coronary artery bypass grafting: a prospective angiographycontrolled study. Eur J Cardiothorac Surg 2011;39:918-23.

13. Niclauss L. Techniques and standards in intraoperative graft verification by transit time flow measurement after coronary artery bypass graft surgery: a critical review. Eur J Cardiothorac Surg 2017;51:26-33.

14. Di Giammarco G, Canosaa C, Foschia M et al. Intraoperative graft verification in coronary surgery: increased diagnostic accuracy adding high-resolution epicardial ultrasonography to transit-time flow

Cite this article as: Kieser TM, Taggart DP. The use of intraoperative graft assessment in guiding graft revision. Ann Cardiothorac Surg 2018;7(5):652-662. doi: 10.21037/ acs.2018.07.06 measurement. Eur J Cardiothorac Surg 2014;45:e41-5.

15. Hiratzka LF, McPherson DD, Brandt B 3rd, et al. Intraoperative high-frequency epicardial echocardiography in coronary revascularization: locating deeply embedded coronary arteries. Ann Thorac Surg 1986;42:S9-11.

16. McPherson DD, Armstrong M, Rose E, et al. High frequency epicardial echocardiography for coronary artery evaluation (in vitro and in vivo validation of arterial lumen and wall thickness measurements). J Am Coll Cardiol 1986;8:600-6.

17. Haaverstad R, Vitale N, Tjomsland O, et al. Intraoperative color Doppler ultrasound assessment of LIMA-to-LAD anastomoses in off-pump coronary artery bypass grafting. Ann Thorac surg 2002;74:S1390-4.

18. Watanabe T, Arai H, Oi K, et al. Detection of internal thoracic artery dissection at coronary anastomosis using intraoperative $15-\mathrm{MHz}$ high-frequency epicardial ultrasound. Circulation 2014;129:e513-5.

19. Iino K, Kato H, Yamamoto Y, et al. Air blockage at coronary anastomosis detected by intraoperative epicardial ultrasonography. Eur J Cardiothorac Surg 2016;50:186-7.

20. Inoue $\mathrm{Y}$, Takahashi R, Tsutsume K, et al. High-frequency ultrasound-guided late surgical revascularisation of chronically occluded left anterior descending coronary artery. Eur J Cardiothorac Surg 2010;37:239-41. 